Cahiers d'études italiennes

12 | 2011

Texte et image dans la culture italienne

\title{
Le vol magique de Messire Torello dans l'édition illustrée du Décaméron (1492, Venise, Gregorio e Giovanni de Gregori)
}

\section{Émilie Zanone}

\section{(2) OpenEdition \\ Journals}

Édition électronique

URL : http://journals.openedition.org/cei/299

DOI : $10.4000 /$ cei.299

ISSN : 2260-779X

Éditeur

UGA Éditions/Université Grenoble Alpes

\section{Édition imprimée}

Date de publication : 15 mars 2011

Pagination : $37-52$

ISBN : 978-2-84310-190-8

ISSN : 1770-9571

Référence électronique

Émilie Zanone, « Le vol magique de Messire Torello dans l'édition illustrée du Décaméron (1492, Venise, Gregorio e Giovanni de Gregori) », Cahiers d'études italiennes [En ligne], 12 | 2011, mis en ligne le 15 septembre 2012, consulté le 27 mars 2021. URL : http://journals.openedition.org/cei/299 ; DOI : https://doi.org/10.4000/cei.299 


\title{
LE VOL MAGIQUE DE MESSIRE TORELLO \\ DANS L'ÉDITION ILLUSTRÉE DU DÉCAMÉRON (1492, VENISE, GREGORIO E GIOVANNI DE GREGORI)
}

\author{
Émilie Zanone \\ Université Stendhal - Grenoble 3, Université de Pise
}

Dans l'avant-dernier récit du Décaméron, Dioneo conte la fabuleuse aventure de messire Torello di Stra da Pavia. Avant de s'enrôler dans les troupes de la chrétienté pour la troisième guerre sainte, messire Torello fait promettre à son épouse de se remarier s'il ne revient pas des combats d'ici un an, un mois et un jour ${ }^{1}$. Lors d'une bataille, le croisé est fait prisonnier par les troupes de Saladin. Il est reconnu par le sultan comme celui qui si courtoisement lui avait offert le gîte et le couvert lors de son voyage incognito en chrétienté. Saladin affranchit le prisonnier et le fait séjourner à sa cour. Mais, la quiétude de Torello est troublée par la nouvelle du naufrage du navire qui transportait la lettre qu'il avait adressée à sa femme pour l'informer de son état. De plus, la mort d'un chevalier du même nom a porté la confusion parmi les gens de Pavie si bien que tous le croient mort. Torello sait que son épouse, fidèle à son engagement, se remariera bientôt. Ne pouvant retourner chez lui rapidement, ce dernier se laisse dépérir. Saladin convoque un magicien qui grâce à son art fait parcourir à Torello la distance entre le Caire et Pavie en une nuit et sur un lit volant. Torello arrive à temps et se fait reconnaître de son épouse.

Le vol magique fonctionne dans le récit en tant qu'expédient narratif dans la mesure où il est un moyen commode de faire avancer l'action. Mais Boccace ne le décrit même pas, préférant s'intéresser aux scènes qui

I. G. Boccaccio, Decameron, X, 9 (éd. V. Branca, Turin, Einaudi, 2005, vol. II, p. I205-I23I). Pour la traduction : Le Décaméron illustré par l'auteur et les peintres de son époque, X, 9 (éd. M. Dozon, C. Guimbard, M. Scialom, revue par C. Bec, Paris, D. De Selliers, 1999, p. 616-628. Toutes les citations en français de la nouvelle de Torello sont tirées de cette édition). 
encadrent cet événement ${ }^{2}$. Il dote ces dernières d'un fort caractère merveilleux suscitant l'imagination, l'étonnement ou le rire si bien que le vol devient un fait marquant du récit tout en étant le grand absent de ce dernier. Souvent, les illustrateurs le choisiront comme un moyen de représenter la nouvelle de manière significative.

La représentation de l'épisode du vol magique est visible dans la première édition italienne imagée du Décaméron, réalisée en 1492 à Venise par les frères imprimeurs De Gregori ${ }^{3}$. Il s'agit d'une xylographie d'un artiste anonyme qui, associé à un compagnon d'œuvre à l'identité incertaine, s'est livré à l'illustration entière de l'édition. Le manuscrit comporte cent quatre xylographies, ou vignettes originales, d'une forme rectangulaire et d'une dimension identique $(57 \mathrm{~mm} \times 73 \mathrm{~mm})$. Le plus souvent, les vignettes se composent de deux parties que la créativité des maîtres graveurs réussit toujours à séparer habilement. Les vignettes accompagnent le texte et chacune met en image un temps de l'action jugé fort, afin de permettre au lecteur d'identifier immédiatement la nouvelle dont il est question. La représentation du voyage de messire Torello remplit partiellement l'espace droit dans le sens de la lecture de la vignette numéro cent onze du tabellaire conservé à la Bibliothèque nationale de Florence. L'autre partie illustre le repas qu'offre Torello à Saladin et à ses troupes.

Cette étude se concentre autour de la représentation du vol magique dans l'édition illustrée du Décaméron des frères De Gregori. Elle consiste à croiser cette dernière avec le texte littéraire, c'est-à-dire avec les passages précédant et suivant le voyage pour montrer les liens entre le texte et l'image. Il s'agit de considérer la subordination et la liberté de la créativité d'un maître graveur par rapport à celle d'un maître écrivain. Ces croisements conduisent également à analyser la façon dont la magie est perçue par les deux auteurs en examinant comment cette pratique, discutée et discutable, est envisagée.

2. Decameron, op. cit., $\$ 76-94$ : «Saladin l'assura que cela allait se faire [...] en le priant de ne pas avoir peur car il était, Torello, son neveu.» (p. 624-625)

3. Exemplaire de la Bibliothèque nationale de Florence, B.R. 365. L'édition De Gregori est la plus ancienne édition italienne illustrée et aussi la plus rare. Ne sont répertoriés que sept exemplaires complets. Pour une description de cette édition voir Prince d'Essling, Études sur l'art de la gravure sur bois à Venise : Les livres à figures vénitiens de la fin du XVe siècle et du commencement du XVt siècle, Florence, Paris, L. Olschki, É. Leclerc, I907-I9I4, 3 vol., vol. I; M. Sander, Le livre à figures italien depuis I465 jusqu'à I530 : essai de sa bibliographie et de son histoire, Milan, Hoepli, 1942, 5 vol., vol. I, p. 187; G. Dillon, «I primi incunaboli illustrati e il Decameron veneziano del I492" dans Boccaccio visualizzato, éd. V. Branca (dir.), Turin, Einaudi, I999, vol. III, p. 29I-3I8; V. Branca, «Le prime illustrazioni» dans Id., Boccaccio medievale, III éd., Florence, Sansoni, I970, p. 322. 


\section{La nouvelle}

Messire Torello réalise en Orient quelques grands rêves de l'homme occidental comme celui de voler ou de parcourir de grandes distances très rapidement. Au Moyen Âge, les voyages représentent des entreprises ardues. Les techniques de transport sont peu avancées, les voies de communications sont précaires, peu entretenues et de nombreux dangers guettent ceux qui les empruntent. La capacité de parcourir en un éclair de grandes distances relève de l'extraordinaire mais, les Orientaux sont, pense-t-on, capables de tous les prodiges. Lorsque Saladin explique à Torello de quelle manière il retournera à Pavie, le narrateur signale que le marchand «ayant entendu dire maintes fois que cela était possible et s'était réalisé commença à se rétablir ${ }^{4} »$. La juxtaposition de la croyance de la réalisation du vol magique et de la convalescence rapide du protagoniste montre qu'il s'agit d'un prodige supposé effectif et commun chez les Orientaux. Dans un même temps, elle indique que les portées de la magie sont positives puisque la santé de Torello s'améliore. Le vol magique a aussi pour effet d'empêcher la polygamie de la dame et de permettre au sultan de rendre à son ami un service aussi noble que celui que le marchand lui avait offert lorsque celui-ci voyageait avec ses troupes incognito en chrétienté.

Les aspects louables et bienveillants de la magie sont l'œuvre d'un magicien "dont il [Saladin] avait déjà expérimenté les talents ${ }^{5}$. Ce nouveau personnage au rôle d'adjuvant dont l'expérience et les succès sont connus du sultan ressort comme un professionnel de la nécromancie. Dans ce récit et plus largement dans le Décaméron, la nécromancie ne désigne pas une pratique de divination par l'invocation des morts ou des démons. Elle est entendue comme un ensemble de connaissances secrètes qui a des répercussions authentiques et vérifiables sur le réel et le modifie. Dans la nouvelle X, 5 , ces connaissances sont celles des lois de la Nature et de ses forces vitales ${ }^{6}$. Elles permettent au magicien de créer un jardin fleuri en hiver. Dans la nouvelle X, 9, elles donnent à ce dernier le pouvoir de contrôler le temps et l'espace. Le puissant somnifère que consomme le futur voyageur n'est pas une œuvre du nécromant, mais celle d'un médecin :

4. "[...] avendo molte volte udito dire che ciò era possibile e fatto s'era assai volte, sincominciò a confortare" (Decameron, op. cit., $\$ 69)$.

5. "[...] a un nigromante la cui arte già espermentata aveva" (ibid., \$70).

6. Ibid., X, 5, \$ IO-I2. Pour les antécédents à cette nouvelle voir les annotations de V. Branca et en particulier note I, p. II48 et note 5, p. II5I. Voir également G. Boccaccio, Filocolo, édité par C. Salinari, N. Sapegno, MilanNaples, Riccardo Ricciardi Editore, I952, p. 850- 852. Voir aussi P. Larivaille (dir.), Dossier Boccace, La nouvelle I0-5 du Decameron, Nanterre, Université Paris Io, 1976. 


\section{Émilie Zanone}

un médecin arriva avec un breuvage et, expliquant à messire Torello que c'était un fortifiant, il le lui fit boire et, un instant après, messire Torello tomba dans un profond sommeil ${ }^{7}$.

L'intervention inopinée d'un pratiquant de la médecine montre que le breuvage n'est pas considéré comme magique. Les frontières entre magie et science sont minces ${ }^{8}$. Les deux pratiques s'appuient sur des lois et sur des causes qui dans les mêmes conditions, devraient produire les mêmes effets ${ }^{9}$. La magie, cependant, est le fruit d'une pensée simple : les cartes de la magie et de la médecine se confondent aisément au XIV ${ }^{e}$ siècle car, la pensée scientifique médiévale n'a pas besoin de rationnel ${ }^{\text {Io }}$. La plupart des médecins pratiquent, par exemple, l'astrologie à des fins curatives. L'attention du narrateur sur le statut du personnage qui tend le breuvage est, à mon avis, significative des limites entendues entre magie et médecine et de la complémentarité de l'une et de l'autre lorsqu'il s'agit de réaliser une activité à la valeur importante. Malinowscki fait remarquer que la magie se lie à la technique lorsque les activités pratiquées font l'objet d'une grande attention et d'une grande perfection technique. Il s'agit pour le magicien d'augmenter le succès de la pratique ${ }^{\text {II }}$.

Le nécromant réalise l'enchantement du lit de manière très rapide. Celui-ci se produit suite aux préparatifs au voyage et aux adieux entre les deux amis. Le devisant, Dioneo, expédie cette action en la réduisant aux seuls mots "exécuter le sortilège" ("[Saladin] dit au magicien d'exécuter sans retard le sortilège $\left.{ }^{\mathrm{I2}} »\right)$. Le contraste entre cette action très brève et les précédentes d'une longueur excessive met en évidence la réalisation rapide

7. "[...] venne un medico con un beveraggio e, fattogli vedere [a Torello] che per fortificamento di lui gliele dava, gliel fece bere; né stette guari che adormentato fu» (Decameron, X, 9, $\$ 84$ ).

8. Les frontières entre magie et science et plus largement entre magie, science et religion font débat chez les anthropologues et les historiens. Plusieurs visions s'opposent. Pour une présentation des débats, voir F. Keck, "Les théories de la magie dans les traditions anthropologiques anglaise et française», Méthodos [en ligne], 2, 2002, mis en ligne le o5 avril 2004. Consulté le 2I novembre 20IO. URL : http://methodos.revue.org/90. Pour davantage de précisions : J. G. Frazer, Il Ramo d'Oro. Studio sulla Magia e la Religione, Roma, Newton Compton, 2006, p. 32-73 (The Golden Bough. A Study in Magic and Religion, London, Macmillan and C, 1936). M. Mauss et H. Hubert, «Esquisse d'une théorie générale sur la magie», Année sociologique, 1902-1903; Levi-Strauss, La pensée sauvage, Paris, Plon, 1966. Pour des analyses critiques de textes de magie médiévales où la frontière entre magie, religion et science est fluctuante voir G. Federici Vescovini, Medioevo magico. La magia tra religione e scienza nei secoli XIII e XIV, Turin, UTET, 2008.

9. J. G. Frazer, «La magia simpatica» dans Il Ramo d'oro, op. cit., p. 32-7I ; M. Mauss et H. Hubert, "Représentation impersonnelles abstraites. Les lois de la magie» dans Magia e scienza., op. cit., p. 39-46.

Io. L. Lallement, "Comment naît une science?» interview à Gérard Simon, Les Cahiers de Sciences et Vie, IO5, 2008, p. I2-I3.

II. B. Malinowscki, Magia, scienza e religione e Baloma. Gli spiriti dei morti nelle isole Trobriand, éd. M. Arioti, Rome, Newton Compton editori, 1976, 202 p. (Magic, science and religion: and other essays); F. Keck, "Les théories de la magie dans les traditions anthropologiques anglaise et française», op. cit., $\$ 8$.

I2. "[...] al nigromante disse [il Saladino] che si spedisse" (Decameron, X, 9, §87). 
de l'enchantement et produit un certain mystère car, celui-ci n'est guère décrit. L'absence de descriptions au sujet de la manière dont la pratique est exercée correspond au secret ordinaire enveloppant l'art occulte. Elle est la démonstration d'un comportement ordinaire des nouvellistes toscans de cette période qui ne décrivent jamais une pratique magique lorsqu'elle se réalise véritablement. Ce silence est aussi dû aux besoins de l'action : Torello doit sans attendre retourner à Pavie. Une description de la pratique ralentirait l'action, d'autant plus que le sultan s'est adonné à une longue décoration du lit et de la tenue du voyageur. La mise en route de l'enchantement relève de la nécessité. Le départ de Torello de la cour du sultan est immédiat et son arrivée soudaine :

[Saladin] dit au magicien d'exécuter sans retard le sortilège. Aussitôt, en présence du sultan, le lit disparut avec messire Torello et Saladin resta là à en parler avec ses dignitaires.

Déjà, dans l'église de San Piero in Ciel d'Oro à Pavie, messire Torello avait été déposé, comme il l'avait demandé, avec tous les joyaux et parures indiqués plus haut ${ }^{13}$.

Le conteur juxtapose les lieux de l'action, si bien que les effets de l'enchantement ressortent comme très rapides et efficaces. D'autres actions de la pratique magique se révèlent au saut du lit de Torello :

ayant ouvert les yeux et regardé autour de lui, [Torello] comprit que de toute évidence il se trouvait là où il avait demandé à Saladin d'être transporté, ce dont il fut fort réjoui. Il se mit sur son séant et, examinant l'une après l'autre toutes les choses qu'il avait autour de lui, bien qu'il connût auparavant la magnificence de Saladin, celle-ci lui parut encore plus grande et il en conçut une plus haute idée ${ }^{\mathrm{I}}$.

Le voyageur se réveille sereinement comme si la nuit précédente avait été ordinaire. Le narrateur décrit son réveil simplement, en hachant chaque étape. Il recourt à des gérondifs qui dilatent les actions et montre ainsi le calme avec lequel ces dernières se déroulent. Les effets de la magie acquièrent une dimension douce et paisible. Cet aspect est aussi renforcé par les scènes qui encadrent le vol. Il s'agit des épisodes des préparatifs au voyage et de la découverte du lit et de son occupant par un petit groupe de moines. Les descriptions des trésors dressent un tableau étincelant des

\footnotetext{
I3. "[...] al nigromande disse [il Saladino] che si spedisse; per che incontanente in presenzia del Saladino il letto e con tutto messer Torello fu tolto via, e il Saladino co'suoi baroni di lui ragionando si rimase. Era già nella chiesa di San Piero in Ciel d'Oro di Pavia, si come dimandato avea, stato posato messer Torello con tutti i sopradetti gioielli e ornamenti" (ibid., \$87-88).

I4. "Messer Torello, aperti gli occhi e da torno guardatosi, conobbe manifestamente sé essere là dove al Saladino domandato avea, di che forte fu seco contento: per che, a seder levatosi e partitamente guardando ciò che da torno avea, quantunque prima avesse la magnificenzia del Saladin conosciuta, ora gli parve maggiore e più la conobbe" (ibid., $\$ 92)$.
} 
innombrables richesses que le sultan a offertes à son ami et dont la beauté rejaillit sur la magie. Les descriptions de celles-ci sont construites par vagues, créant un crescendo de la splendeur des préparatifs. Le narrateur décrit d'abord le lit et Torello. Puis, il suspend la description pour avancer l'action mais, il revient très vite à la description des préparatifs. Comme étourdi par les trésors, il décrit à la fois ceux qui sont ajoutés au meuble et à la tenue du voyageur. Un parallèle est possible entre le comportement du narrateur et du sultan. Tous deux semblent incontrôlables devant les richesses. Saladin accumule ses dons jusqu'à provoquer l'impatience du magicien. Il ne se retient que devant l'irritation de celui-ci et l'urgence de réaliser l'enchantement. Le narrateur cesse avec difficulté de parler des ors, des pierres précieuses ou des rares tentures. Celui-ci se laisse emporter par les mots, comme fasciné et excité par la somptuosité des présents. C'est avec des yeux écarquillés que le lecteur et la brigata découvrent la profusion d'or, d'argent, de gemmes et de tissus précieux. Par exemple, Saladin

fit dresser dans une grande salle un superbe et riche divan à la mode du pays, avec des matelas de velours et de soieries brodés d'or, et il fit poser par-dessus une couverture ornée d'arabesques en très grosses perles et en joyaux de grand prix, qui fut par la suite considérée en Occident comme un trésor infiniment précieux, ainsi que deux oreillers qui convenaient à un tel lit ${ }^{15}$.

Boccace use et abuse d'un lexique marqué par la préciosité. Le meuble est verni de luxueux accessoires liés les uns aux autres par une longue énumération. Chaque élément est richement qualifié par le biais d'adjectifs qualificatifs ou de groupes nominaux compléments du nom. Des couvertures aux tissus précieux ornés de riches motifs parent la couchette volante. Celle-ci possède des dimensions exceptionnelles car, la pièce où elle est installée est grande. Le meuble est garni de larges matelas et de confortables oreillers. Les grandes dimensions et les accessoires font du lit qui est déjà en lui-même un signe de richesse ${ }^{16}$, un meuble noble et hors du commun. La noblesse de ce mobilier s'accorde avec celle de son occupant. Messire Torello devient un sultan des mille et une nuits. Il est revêtu "d'une robe à la mode sarrasine, la plus belle et la plus riche qui ait jamais été ${ }^{17}$ » et il porte autour de la tête un turban appartenant à

\footnotetext{
I5. "[...] fece il Saladin fare in una gran sala un bellissimo e ricco letto di materassi, secondo la loro usanza, tutti di velluti e di drappi ad oro, e fecevi por suso una coltre lavorata a certi compassi di perle grossissime e di carissime pietre preziose, la qual fu poi di qua stimata infinito tesoro, e due guanciali quali a cosi fatto letto si richiedeano" (ibid., $\$ 76-77$ ).

I6. C. Dubord, «Le lit : histoire et formes», Meuble peint, disponible sur http://www.meublepeint.com/ histoire-lits.htm

I7. "[...] una roba alla guisa saracinesca, la più ricca e la più bella cosa che mai fosse stata veduta per alcuno" (ibid., $\$ 77)$.
} 
Saladin ${ }^{18}$. Il devient ainsi l'égal du souverain musulman ${ }^{19}$. La beauté de chacun des atours est magnifiée par l'emploi de tournures superlatives et marquant l'exceptionnalité. Une fois endormi, Saladin offre au voyageur des présents dignes des seigneurs que le narrateur insère dans une longue et précieuse énumération ${ }^{20}$. Torello reçoit "une haute et belle couronne de grand prix [...] une bague ornée d'une escarboucle [ou encore] une épée». La noblesse des atours de Torello reflète celle de son cœur puisque le marchand s'est illustré pour sa magnanimité. Chaque présent de Saladin resplendit d'éclat et leur nombre est si grand que Dioneo renonce à les énumérer. Tout se déroule dans le faste absolu. Cette profusion d'or et de métaux précieux, d'inestimables étoffes et de rares joyaux confère à la scène des préparatifs une forte tonalité merveilleuse.

Le faste des trésors accorde à la magie une nature merveilleuse qui est, cependant, marquée par le concret. L'extraordinaire y est rationnel. Les descriptions sont truffées d'indications marchandes. La parure du lit est faite d' « une couverture ornée d'arabesques en très grosses perles et en joyaux de grand prix, qui fut par la suite considérée en Occident comme un trésor infiniment précieux ${ }^{2 \mathrm{I}}$ ». Torello est vêtu d'une tunique «la plus belle et la plus riche qui ait jamais été vue ${ }^{22}$ » et il a enfilé au doigt une bague «d'une valeur pour ainsi dire inestimable ${ }^{23} »$. L'ornementation de l'épée est "d'une richesse difficile à évaluer ${ }^{24}$ » et la broche est faite de perles «comme on n'en avait jamais vues ${ }^{25}$ ». La valeur inestimable sublime les dons mais, la

\footnotetext{
I8. "[Saladino] commandò che a messer Torello [...] in testa alla loro guisa una delle sue lunghissime bende [fosse messa] a ravolgere"; "et autour de la tête [Saladin] lui fit [à Torello] enrouler un de ses longs rubans" (ibid., $\$ 77)$.

19. Lors de l'épisode durant lequel Saladin propose à Torello de regagner Pavie sur un lit volant, il lui avoue son regret de le voir partir car, il formulait le projet de partager avec lui son royaume: "Sarebbemi stato carissimo [...] che quel tempo, che voi e io viver dobbiamo, nel governo del regno che io tengo parimente signori vivuti fossimo insieme»; "j'aurais bien aimé que nous passions ensemble le temps qu'il nous reste à vivre, tous deux gouvernant de pair mon royaume" (ibid., $\$ 73$ ).

20. "[...] una grande e bella corona [pose Il Saladino] di gran valore e si la segnò, che apertamente fu poi compreso quella dal Saladino alla donna di messer Torello esser mandata. Appresso mise in dito a messer Torello uno anello nel quale era legato un carbuncolo tanto lucente, che un torchio acceso pareva, il valor del quale appena si poteva stimare; quindi gli fece una spada cignere il cui guernimento non si saria di leggieri apprezzato; e oltre a questo un fermaglio gli fé davanti appiccare nel quale erano perle mai simili vedute con altre care pietre assai»; "le sultan posa à côté de lui une haute et belle couronne de grand prix et la marqua de telle sorte qu'ensuite on comprit parfaitement qu'elle était envoyée par Saladin à l'épouse de messire Torello. Après cela, il mit au doigt de celui-ci une bague ornée d'une escarboucle si flamboyante qu'elle avait l'air d'une torche enflammée, d'une valeur pour ainsi dire inestimable; puis il lui fit une ceindre une épée dont l'ornementation était d'une richesse difficile à évaluer; en outre, il fit agrafer sur son vêtement une broche garnie de perles précieuses" (ibid., \$ 86).

2I. "[...] una coltre lavorata a certi compassi di perle grossissime e di carissime pietre preziose, la qual fu poi di qua stimata infinito tesoro" (ibid., $\$ 76$ ).

22. "[...] la più ricca e la più bella cosa che mai fosse stata veduta per alcuno" (ibid., \$ 77).

23. "[...] il valor del quale [ dell’anello] appena si poteva stimare» (ibid., \$ 86).

24. "[...] non si saria di leggieri apprezzato" (ibid., $\$ 86$ ).

25. "[...] mai simili vedute» (ibid., $\$ 86$ ).
} 
tentative de les évaluer les rationalise en les ramenant à une valeur commerciale et concrète. Le réalisme se manifeste aussi à travers la réaction très humaine de peur du sacristain. Cette scène tire son caractère comique d'un renversement de l'étonnement. Le lecteur a admis le caractère exceptionnel du lit et de son voyageur. La surprise, la peur et la fuite des moines ressortent comme injustifiées et imprévues. Dans la logique du récit, elles sont étonnantes alors que d'ordinaire l'inverse se produirait. La réaction des moines est aussi comique du fait que le lecteur a une connaissance des événements supérieure à celle de ces derniers. Un décalage se crée et leur réaction est perçue comme saugrenue. Le comportement de l'abbé fait sourire, en particulier. Il rassure le sacristain et il se moque de sa peur injustifiée. Mais, lorsqu'il se trouve devant Torello endormi, il perd ses moyens et s'écrie : «Dieu, aide-nous ${ }^{26}$ !» avant de prendre ses jambes à son cou. La richesse du lit interloque les religieux mais de manière comique, ce qui montre que la magie opérée est bien peu dangereuse.

La préparation du vol magique et l'arrivée du voyageur dans la basilique de San Pietro in Ciel d'Oro constituent une pause narrative dans laquelle Boccace se laisse émerveiller et émerveille son lecteur. Il requiert la mise en œuvre d'une magie orientale, bienveillante et douce en supprimant tout aspect démoniaque. Il en fait un ensemble de connaissances permettant de maîtriser le temps et l'espace. La magie apparaît comme une alliée efficace des protagonistes les aidant à la réalisation de nobles causes. Bien que Boccace taise la réalisation du vol, il suggère aisément sa douceur et son caractère fabuleux en se laissant emporter par le faste des préparatifs. À l'arrivée de Torello dans la sacristie, il court faire partager la surprise du sacristain et provoque une scène comique qui confère à la magie un caractère inoffensif.

\section{La xylographie}

La xylographie de l'édition des frères De Gregori met en scène l'entrée imminente du lit volant et de son occupant à l'intérieur de l'église San Pietro in Ciel d'Oro. De cette image émane une grande simplicité. Les éléments naturels, architecturaux et humains sont rendus visiblement dans un style pauvre, de "manière presque abréviative» ainsi que l'affirme Dillon dans sa présentation des images du tabellaire ${ }^{27}$. Cette simplicité

26. "Domine, aiutaci.» (Ibid., \$91.)

27. G. Dillon, «I primi incunabuli» dans Boccaccio visualizzato, op .cit., p. 315. 
serait due à la difficulté de la gravure sur bois réalisée à l'aide de moyens techniques rudimentaires et à la nécessité de représenter une image dans une vignette aux petites dimensions. Il s'agirait aussi de l'expression stylistique propre à l'artiste. En effet, les vignettes gravées de sa main présentent un style dépouillé de tout ornement.

Cette simplicité se cueille au second plan de l'image du vol où un paysage de campagne en friche est représenté. De grossiers traits verticaux et légèrement inclinés suggèrent des herbes hautes. Certains, auréolés de formes arrondies et étirées indiquent des arbustes. La végétation éparse reste discrète dans la mesure où elle est de petites dimensions. Celle-ci est comme posée maladroitement à la cime de vallons, marquant la frontière entre le ciel et la terre. Ces reliefs sont représentés par un trait simple et ondulé. Quelques autres traits furtifs suggèrent des reliefs moins élevés. Ils évitent que l'espace entre les vallons du second plan et l'église du premier plan soit vide. Au centre du second plan, une construction rompt la monotonie du triste paysage. C'est une humble construction dont le rôle n'est pas identifiable. Il s'agit peut-être de la demeure des futurs époux où Torello se rendra pour empêcher les noces ou de celle de ce dernier qu'il regagnera à la fin du récit ${ }^{28}$. Le bâtiment se situe au creux de deux vallons sans donner, tout comme ces reliefs, de la profondeur à l'image. La scène représentée à ciel ouvert et le décor naturel montrent qu'elle se déroule dans le contado, loin de la cité et de sa foule. Le xylographe prend une liberté par rapport à la réalité dans la mesure où l'église de San Pietro in Ciel d'Oro est située au centre de la Pavie médiévale. Il adapte l'image au besoin de représenter une scène dans un espace restreint et évite un décor urbain ou d'intérieur aux nombreux détails. Il s'agit aussi d'une invention par rapport au texte littéraire dans la mesure où, Boccace situe les scènes pré et post voyage en intérieur.

Dans le dépouillement de l'image, le xylographe va même jusqu'à négliger un lieu clé de l'action littéraire, c'est-à-dire l'église. L'édifice est très partiellement représenté au premier plan. Seule une petite partie de sa façade est visible et elle est privée de tout ornement. Aucun de ses célèbres éléments architecturaux n'est présent. Le graveur oublie les précieux motifs de la façade ou les trois fameux arcs-boutants qui encadrent l'entrée de l'édifice et qui en font la renommée. Seule une vulgaire ouverture en forme de demi-cercle agrémente la façade. Au-dessous de cette ouverture se trouve l'entrée du

28. Le protagoniste "dans le costume qu'il portait, se rendit avec l'abbé chez le marié» et une fois son identité révélée, avec son épouse, il s’en retourne chez lui : "Sortis de la maison où ils étaient, avec toute la grande pompe des noces, ils allèrent à celle de messire Torello.» 
bâtiment. Elle se présente sobrement sous la forme d'un rectangle dont la hauteur est grande. Seule une partie est visible et elle est noircie afin d'indiquer l'intérieur de l'édifice. Il est impossible d'identifier l'église San Pietro in Ciel d'Oro sans connaître le récit, ce qui subordonne immédiatement l'image au texte. Perpendiculaire à l'entrée de l'église, un tombeau est, peut-être, représenté. Il s'agit d'un caisson situé dans la partie inférieure de l'image et portant deux abréviations latines. La première est " $\mathrm{SEP}^{29}$ » et elle signifie "sépulcre». "La seconde abréviation se trouve en dessous et correspond à «OIO». D'après le Lexicon abbreviaturarum, il existe la forme "OIO » auréolée d'un accent circonflexe largement ouvert indiquant la suppression par contraction d'une lettre intermédiaire ${ }^{30}$. Il s'agit de la lettre «m». $\mathrm{La}$ forme complète de "OIO» est "omino» qui signifie «tout». Notons, d'ailleurs que le Lexicon date l'abréviation de la fin du $\mathrm{xv}^{\mathrm{e}}$ siècle, soit de la même époque que le tabellaire. Par cette abréviation, le graveur souhaite, je pense, indiquer le "Seigneur» car il est tout. Les inscriptions ne sont garnies d'aucun ornement et leurs caractères sont formés simplement. Il n'est guère aisé de comprendre le lien entre ces inscriptions et le reste des éléments de l'image, ni même la présence et la fonction de ce caisson. Celui-ci possède des dimensions disproportionnées par rapport à l'édifice et il se situe en dessous du lit de messire Torello, qui est l'élément clé de l'image. L'œil du spectateur cueille cet élément immédiatement, ce qui attribue à ce dernier une certaine importance. Cependant, les trop grandes dimensions de ce caisson confèrent un aspect maladroit à l'image; en opposition totale au raffinement du texte. Il s'agit, sans doute, d'un signe indiquant la nature et la fonction du bâtiment dont le sens s'est aujourd'hui perdu. L'église de San Pietro in Ciel d'Oro est célèbre pour abriter les reliques de saint Augustin. Reconnaitre, cependant, le tombeau de l'un des pères fondateurs dans ce caisson se révèle expéditif, hasardeux et bien téméraire.

$\mathrm{Au}$-dessus du présumé tombeau se trouvent le lit magique et son occupant. Ces derniers éléments remplissent la partie supérieure du premier plan de l'image. Le meuble est de grandes dimensions. Il remplit un ciel dépourvu de nuages et d'astres. L'artiste le situe avec maladresse car, il le place sur le même plan que l'entrée de l'église. L'absence de perspective produit un effet de déséquilibre comme si, le meuble s'apprêtait à percuter la façade. Cette disposition accentue le caractère populaire de l'image qui est renforcée par la suppression de toutes les richesses dont est paré

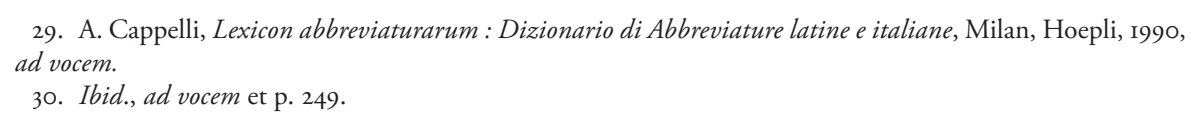


le lit à la cour de Saladin. Le graveur réduit le magnifique meuble, orné richement et patiemment par le sultan, à un lit des plus sobres et simples. L'artiste n'annule pas entièrement le faste du texte littéraire puisqu'il dépose une couronne sur les couvertures. Il s'agit d'une métonymie visuelle des innombrables trésors. La couronne symbolise la royauté, le pouvoir et surtout la richesse. Elle concentre à elle seule les longues descriptions des trésors avec lesquels messire Torello gagne l'Occident. Elle rappelle aussi aux spectateurs les raisons du vol car, elle est à l'intention d'Adelaïde, l'épouse de Torello. Boccace choisit également la couronne pour rappeler les innombrables richesses que le croisé rapporte d'Orient.

Torello est représenté endormi, allongé sur le lit. Son visage n’a aucune expression du fait de son état. Son corps est caché sous les couvertures. Celles-ci se révèlent être un moyen commode d'éviter de représenter la tenue "à la mode sarrasine» de Torello. Elles évitent au graveur de réaliser trop de détails. Seuls la tête, le buste et une main du personnage sont visibles. Torello ne se présente pas sous les traits d'un prince arabe. Il est coiffé d'un simple bonnet qu'il porte déjà dans la première partie de la xylographie mettant en image le banquet qu'il donne en l'honneur de Saladin. Ce chapeau est un signe distinctif du personnage : il permet de le reconnaître de manière immédiate et de lier ensemble la première et la deuxième partie de la xylographie.

Torello voyage sur le lit, poussé par des diables. Chacune de ces trois créatures qui pousse le lit se situe à un des pieds de celui-ci, si bien qu'on peut se douter de la présence d'une quatrième créature. Il faut chercher dans le nombre de ces diables des raisons pratiques, c'est-à-dire celle de porter un lit imposant à plusieurs et celle d'obtenir une certaine stabilité. Ces diables se présentent sous les traits typiques des créatures infernales de l'époque médiévale. Leur apparence est laide afin de mieux susciter la peur. Leur corps est décharné, nu et dépourvu de pelage ou de plumage. Leurs griffes sont acérées et leur nez crochu. Leur apparence est peu soignée et aucun ne manifeste d'expression. L'œil du spectateur les repère rapidement et aisément, car les créatures diaboliques se trouvent au centre du premier plan. Elles représentent de manière visible ${ }^{3 \mathrm{I}}$ la magie. Il s'agit d'une invention de l'artiste-graveur qui se place en totale opposition

3I. Nous pouvons penser au Giudizio universale de Giotto sur la façade intérieure de la Cappella degli Scrovegni (I303-I305) de Padoue ou au Trionfo della morte (I336-I34I) de Buonamico Buffalmacco à Pise. Pour des études critiques sur l'iconographie du diable : Enciclopedia dell'arte medievale, Rome, I99I-2000, I2 vol., vol. V, ad vocem; J. Baschet, "Satan ou la majesté maléfique dans les miniatures de la fin du Moyen Âge», dans Le mal et le diable : leurs figures à la fin du Moyen Âge, N. Nabert (dir.), Paris, Éditions Beauchesne, I996, p. I87-210; Diables et diableries : la représentation du diable dans la gravure des XV et XVI siècles, F. Rodari et J. Wirth (dir.), Genève, I977. 
avec le texte de Boccace, cependant la représentation de la magie sous la forme de pratique diabolique n'est pas une invention originale de la part de l'artiste.

\section{La représentation diabolique de la magie : quelques pistes de réflexion}

Nous proposons deux pistes de réflexion sur les créatures diaboliques. Une première piste considère leur présence comme un témoignage sur la perception de la magie à la fin du Xv $v^{\mathrm{e}}$ siècle. La vignette est élaborée dans une société chrétienne qui envisage la magie comme un art du diable ${ }^{32}$. Les pères de l'Église, saint Augustin en tête, identifient la magie comme une pratique des mauvais esprits, appelés "daimones ${ }^{33}$ ». Il s'agit des anges qui ont soutenu Lucifer dans sa rébellion contre Dieu et qui l'ont suivi dans sa chute ${ }^{34}$ ou des divinités païennes déclassées au rang de démon. Pour l'évêque d'Hippone

[...] les démons aiment bien les malpropretés de la scène, et la pudeur ne l'apprécient point; les démons savourent, dans les manigances des magiciens, les mille façons de nuire que n'aime pas l'innocence ${ }^{35}$.

En exerçant l'art occulte, le magicien devient un disciple des démons. Il croit réaliser toutes sortes de prodiges que les simples capacités humaines ne permettent pas d'accomplir. Il rentre en conflit avec le principe fondateur du dieu unique et se rend coupable d'idolâtrie et d'apostasie. La prétention de l'officiant en magie de dépasser le Seigneur est illustrée plus d'une fois dans la Bible qui est très intolérante face à l'art occulte. Un des célèbres épisodes de condamnation de la magie est celui de l'affrontement entre Simon et saint Pierre. Les Actes des Apôtres ${ }^{36}$ rapportent que le magicien Simon se convertit au christianisme, ébloui par les prodiges du diacre Philippe. Mais lorsqu'il voit les apôtres Pierre et Jean donner le Saint-Esprit, il demande à pouvoir le faire, même moyennant de l'argent. Indigné, Pierre lui répond :

\footnotetext{
32. F. Cardini, Magia, stregoneria e superstizione nell'Occidente medievale, Florence, La Nuova Italia, I979. 33. Saint Augustin, La Cité de Dieu dans Euvres, II, L. Jerphagnon (dir.), Paris, Gallimard, 20oo. Voir par exemple, livre VIII, chap. XVI, p. 3 I7 «La démonologie d'Apulée» : "Ce sont eux, précise-t-il [Apulée] entre autres choses, qui s'occupent des prédictions des augures, haruspices, devins, et de celles qu'apportent les songes, et enfin des prodiges accomplis par les mages "; voir aussi livre VIII, chap. XVIII.

34. Isaïe I4, I2- I5; Luc IO, I8.

35. Saint Augustin, La Cité de Dieu, op. cit., livre VIII, chap. XviıI, p. 320.

36. Actes des Apôtres, VIII, 9.
} 
Que ton argent périsse avec toi, puisque tu as cru que le don de Dieu s'acquérait à prix d'argent!/ Il n'y a pour toi ni part ni lot dans cette affaire, car ton cœur n'est pas droit devant Dieu./ Repens-toi donc de ta méchanceté, et prie le Seigneur pour que la pensée de ton cœur te soit pardonnée, s'il est possible;/ car je vois que tu es dans un fiel amer et dans les liens de l'iniquités ${ }^{37}$.

La faute de Simon est celle de vouloir acheter un bien spirituel pour un prix temporel. De celle-ci, dérive la simonie. L'épisode biblique punit plus cette faute que celle de la pratique de la magie. Cependant, il établit un lien entre les deux erreurs dans la mesure où, ces dernières renforcent la nature exécrable de Simon. La magie pousse celui-ci à croire qu'il peut tout obtenir sans aucune crainte et être l'égal du Seigneur. Elle fait de lui un être plein de mépris, de prétention et d'arrogance; attitudes insupportables dont seront caractérisés les magiciens. Dans les Actes de Pierre, Simon affronte à nouveau saint Pierre. Le magicien n'a rien perdu de sa superbe. Simon enchante les foules romaines par ses prodiges. Pierre qui se trouve à Jérusalem a une révélation dans laquelle il voit les prodiges du magicien. Le Christ l'envoie à Rome pour combattre Simon. Pierre réalise plusieurs miracles qui, loin d'intimider le magicien le font persister dans son erreur jusqu'à annoncer qu'il s'envolera vers Dieu. Pierre implore le Seigneur de ne pas laisser faire le magicien. Simon s'envole mais, il s'écrase sur le sol et est lapidé par la foule ${ }^{38}$. Bien que tiré d'un texte apocryphe controversé, cet épisode montre toute l'hostilité de la religion chrétienne contre la pratique occulte.

Cette animosité contre la magie est particulièrement palpable à la fin du Xv $v^{\mathrm{e}}$ siècle. La préparation et l'impression de l'édition De Gregori appartiennent à une période de troubles et de peur face à la magie et au démon. Durant tout le $\mathrm{XIV}^{\mathrm{e}}$ siècle, l'Église met en ouvre un arsenal doctrinal et inquisitorial afin de lutter contre la magie et le diable ${ }^{39}$. Celui-ci arrive

\footnotetext{
37. Ibid., 20-23.

38. Actes de Pierre : "Simon, se tenant debout sur un lieu élevé et regardant Pierre, se mit à dire : - Pierre, maintenant que je m'élève sous les yeux de tous ces spectateurs, je te le dis : si ton dieu est puissant, lui que les juifs ont mis à mort - et ils vous ont lapidés, vous qu'il avait choisis -, qu’il prouve que la foi en lui est la foi en Dieu, que soit clair maintenant si elle est digne de Dieu. Car moi, en m'élevant, je ferai voir à toute cette foule qui je suis. Et voilà qu'il s'éleva dans les airs, tout le monde le voyait de tout Rome, élevé au-dessus de ses temples et de ses collines; les croyants, eux, détournaient les yeux vers Pierre. Et Pierre, à la vue de ce spectacle inouï, cria vers le Seigneur Jésus en disant : - Si tu laisses celui-ci faire ce qu'il a entrepris, alors tous ceux qui ont cru en toi seront scandalisés, et les signes et prodiges que tu leur as accordés par moi ne seront plus dignes de foi. Vite, Seigneur, montre ta grâce : que, tombant des airs, il ressente une extrême faiblesse, qu'il ne meure pas, mais qu’il soit épuisé et se brise la jambe en trois endroits. Et, tombant des airs, il se brisa la jambe en trois endroits. Alors, on le lapida, puis chacun rentra chez soi, tous désormais ayant foi en Pierre.» (Publié dans R. Gounelles, "Lire dans les textes apocryphes chrétiens", Cahier des Évangiles, I48, p. I09-IIo.)

39. L'instauration de cet arsenal est complexe et est constituée de plusieurs étapes longues qui sont à l'initiative d'acteurs différents. Voir S. Abbiati, A. Agnoletto, M. R. Lazzati, La stregoneria : diavoli, streghe,
} 
à maturation à la fin du $\mathrm{XV}^{\mathrm{e}}$ siècle. En I486, les dominicains Sprenger et Institoris publient le grand manuel inquisitorial, le Malleus Maleficarum ${ }^{40}$ dans le but d'aider les inquisiteurs dans la lutte contre la prolifération des officiants de l'art occulte. Placée en introduction au manuel, la bulle du pape Innocent VIII, Summis desiderantes affectibus officialise et rend légitime les propos des inquisiteurs, les encourage et exhorte les chrétiens à se lancer dans l'extermination des sorcières et magiciens. Le zèle des inquisiteurs, la peur de l'invasion des disciples du démon dans le royaume de Dieu, l'imprimerie et le format in octavo du manuel facilitent la circulation des idées anti-magiques et leur consultation. Rapidement, de grandes chasses aux sorcières se déroulent dans toute l'Europe.

Le graveur de la xylographie du vol magique baigne dans ce climat de tension et de lutte, tout comme son public. Afin que le lien entre l'image et le texte, écrit plus d'un siècle auparavant soit immédiat, le graveur se doit de recourir à des signes dont il partage le sens avec son public. Son choix de faire de la magie une pratique du diable et de représenter de telles créatures pour faire voyager le lit est significatif du contexte idéologique de l'époque.

La seconde hypothèse qui expliquerait la présence des diables au pied du lit du Torello considère la diffusion du motif du diable comme initiateur du voyage extraordinaire dans la littérature européenne de cette époque. Les travaux sur les sources du Décaméron ont montré que la nouvelle de messire Torello s'inscrit dans un ensemble de récits populaires où des vols magiques similaires se produisent grâce au diable ${ }^{4 \mathrm{I}}$. Des faits analogues se retrouvent dans l'Avventuroso Ciciliano ${ }^{42}$, dans le Conde Lucanor $^{43}$ et dans de nombreux textes issus de la culture germanique. Pio Rajna, puis Michele Landau et plus récemment Carlo Delcorno identifient l'épisode

\footnotetext{
inquisitori dal Trecento al Cinquecento, Milan, Mondadori, 1984; A. Boureau, Satan hérétique. Naissance de la démonologie dans l'Occident médiéval (I280-I330), Paris, Odile Jacob, 2004; voir aussi le compte rendu de cet ouvrage par M. Ostorero, "A. Boureau, Satan hérétique. Naissance de la démonologie dans l'Occident médiéval (I280-I330)", Medievales [en ligne], 48, printemps 2005, mis en ligne le 02 décembre 2005. Consulté le 2I novembre 20Io. URL : //medievales.revues.org/I087; J. P. Boudet, "Les condamnations de la magie à Paris en I398", Revue Mabillon, I2, 200I, p. I2I-I57; F. Mormando, The Preacher's Demons: Bernardino of Siena and the Social Underworld of the Early Renaissance Italy, Chicago-Londres, The University of Chicago Press, I999, p. 52-108.

40. H. Institoris et J. Sprenger, Malleus Maleficarum. Le marteau des sorcières, éd. A. Danet, Grenoble, J. Millon éditions, 1999 et A. Danet, L'inquisiteur et ses sorcières dans H. Institoris et J. Sprenger, op. cit., p. II-94.

4I. P. Rajna, "La novella boccaccesca del Saladino e di messer Torello», Romania, 6, I877, p. 359-368; M. Landau, "La novella di messer Torello (Decam., X, 9) e le sue attinenze mitiche e leggendarie», Giornale Storico della Letteratura Italiana, 2, I883, p. 59-I08.

42. Sur les liens entre le Décaméron et l'Avventuroso Ciciliano voir : R. Gigliucci, "Argumentum, historia : nota su Avventuroso siciliano e Decameron", Studi sul Boccaccio, 23, 1995, p. 245-253.

43. L. Russo, Letture critiche del Decameron, Bari, Laterza, 1956.
} 
du vol magique de Boccace à celui qu'effectue le chevalier allemand Gérard dans le Dialogus Miraculorum du frère cistercien Césaire de Heisterbach $(\dagger \mathrm{I} 240)^{44}$. L'exemplum met en scène un chevalier prénommé Gérard et dévot au culte de saint Thomas. Celui-ci part en pèlerinage aux Indes pour honorer le saint. Avant son départ, il demande à son épouse de se remarier s'il n'est pas de retour d'ici cinq ans: "Je te demande d'attendre cinq ans mon retour, après cela, épouse qui tu veux ${ }^{45}$." Aux Indes, Gérard vit de prières et oublie sa promesse jusqu’à la veille de l'échéance donnée à son épouse. Le diable lui apparaît et lui dit qu'il a pour mission de le ramener en Allemagne :

Je suis le diable, et il m’a été demandé, avant que les gens aillent dormir, de te ramener chez toi parce que ta femme s'apprête à se marier avec un autre homme, et elle est en ce moment déjà assise avec lui au banquet nuptial. Il le prit, et en moins d'une journée, il le transporta des Indes à l'Allemagne, de l'Orient à l'Occident, et vers le crépuscule il le déposa dans son palais sain et sauf ${ }^{46}$.

Le Dialogus Miraculorum remontre à i222. Il est probable qu'il circule en Italie et pendant très longtemps. Le graveur de l'édition De Gregori connaît, peut-être, ces sources et s'en est inspiré.

Le croisement de la vignette cent onze de la première édition italienne illustrée du Décaméron et des épisodes pré- et post-voyage de la nouvelle $\mathrm{X}, 9$ révèle la capacité créatrice d'un maître graveur dont la mission est de rendre visible ce que Boccace a tu en le reliant au texte de sorte que celui-ci soit identifiable immédiatement. L'artiste imagier subordonne sa créativité au récit en reprenant les éléments fondamentaux comme l'église, le lit volant et Torello. Mais il se libère aussi franchement du Décaméron en aplatissant la scène. Il est avare de détails, de richesses et de personnages jusqu'à finir par mettre en opposition l'image et le texte. Le raffinement et la richesse du récit sont contrecarrés par un style rustre et populaire. Cette pauvreté est due à des exigences techniques : les outils de gravure sont élémentaires et l'espace de la vignette est réduit. Cette simplicité s'explique par une raison stylistique : elle est la griffe de l'illustrateur. Elle

44. Une version en ligne du Dialogus Miraculorum édité par J. Strange est disponible sur le site du GAHOM : http://gahom.ehess.fr/document.php?id=72I. Pour de plus amples informations sur Césaire de Heisterbach, voir : Césaire en ligne disponible sur le site du GAHOM : http://betula.annexus.ehess.fr/sdx/cesaire/index.xps. 45. La traduction est nôtre. Texte original : "Rogo etiam ut quinque annis reditum meum expectes; quibus expletis, nubas cui volueris."

46. La traduction est nôtre. Texte original : «Ego sum diabolus, et praeceptum est mihi, ut antequam homines cubitum vadant, in domum tuam te transferant, eo quod uxor tua alteri viro nupserit, et iam in nuptiis cum illo sedeat. Tollens eum, in parte ab India in Theutoniam, ab ortu solis in eius occasum transverit, et circa crepusculum in curia propria illum sine laesione deposuit." 
est enfin idéologique car elle attire immédiatement l'œil du spectateur sur les créatures diaboliques.

Les créatures démoniaques incarnent la magie. La présence des diables est la majeure innovation du maître et trouve son origine soit dans les traditions littéraires qui font du diable un promoteur des voyages fantastiques soit dans la conception chrétienne de la magie. L'art occulte y est vu comme une œuvre du diable. La diffusion de cette conception est particulièrement forte à la fin du $\mathrm{Xv}^{\mathrm{e}}$ siècle.

L'image ne joue donc pas seulement le rôle de représenter un texte. Elle est un témoignage des mentalités et des motifs littéraires traditionnels de source folklorique. Quant au jugement de la magie chez Boccace, nous pensons qu'il ne faut pas rechercher dans la réalisation du vol le reflet d'une croyance à une magie authentique aux effets vérifiables. Dans la nouvelle X, 9 comme dans la nouvelle X, 5, la magie appartient au merveilleux et elle se réalise pour cela. Elle est bien différente de celle des nouvelles VIII, 3 ou IX, 5 qui racontent les aventures de Calandrino où le pauvre bougre se fait moquer pour croire qu'un vulgaire caillou ou qu'un banal bout de parchemin puisse être magique. Pour Boccace, la magie est ailleurs : elle est l'œuvre de nécromants orientaux, elle s'exerce dans des terres lointaines où tout est possible. 